\title{
Excitation-energy sorting in superfluid fission dynamics
}

\author{
B. Jurado ${ }^{\mathrm{a}}$ and K.-H. Schmidt ${ }^{\mathrm{b}}$ \\ CENBG, CNRS/IN2P3, Chemin du Solarium, BP. 120, 33175 Gradignan, France
}

\begin{abstract}
It is now well established that at moderate excitation energies the nucleus temperature does not vary with increasing excitation energy. We show that, as a consequence, two nuclei with different temperatures brought into contact show a rather surprising energy-sorting mechanism where the hotter nucleus transfers all its excitation energy to the colder one. The scission configuration of the fission process offers a unique possibility to observe this phenomenon. The energy-sorting mechanism is clearly reflected by the mean number of prompt neutrons as a function of the fragment mass and by the dependence of the local even-odd effect with mass asymmetry.
\end{abstract}

\section{Introduction}

Experimental data on nuclear level densities [1] show that the behavior of nuclei at moderate excitation energies $E^{*}$ is well described by a constant-temperature level density of the form:

$$
\rho\left(E^{*}\right) \propto \exp \left(\frac{E^{*}}{T}\right)
$$

where $\mathrm{T}$ is the constant temperature parameter. This means that in this regime the temperature of nuclei does not vary with $E^{*}$. The main reason for this constant-temperature behavior is that pairing correlations lead to an effective number of degrees of freedom that increases in proportion to $E^{*}$. Cooper pairs of neutrons and protons melt in a way that the mean energy per nucleonic excitation and thus the nuclear temperature stays constant. In nature, this behavior appears in first-order phase transitions (e.g., solid-liquid or liquid-gas). In a mixture of two phases, such as ice and water, the temperature of the mixture remains constant when energy is introduced or extracted, as long as both phases are present. Only the fractions of the two phases vary. In a recent experimental work on level densities [2] it has been surprisingly found that the constant-temperature behavior remains up to an excitation energy of $20 \mathrm{MeV}$. In Ref. [1] the authors fitted expression (1) to experimental data on level densities for nuclei ranging from ${ }^{18} \mathrm{~F}$ to ${ }^{251} \mathrm{Cf}$. The following dependence of the constant temperature $T$ with the mass $A$ of the nucleus and the shell correction $S$ was found:

$$
T=A^{\frac{-2}{3}}\left(17.45-0.51 S+0.051 S^{2}\right)
$$

In this work we investigate how two nuclei in the particular regime of constant temperature behave when they are set in thermal contact. This situation is realized near the scission configuration in fission.

\footnotetext{
a e-mail: jurado@cenbg.in2p3.fr

b e-mail: k.h.schmidt@gsi.de
}

This is an Open Access article distributed under the terms of the Creative Commons Attribution-Noncommercial License 3.0, which permits unrestricted use, distribution, and reproduction in any noncommercial medium, provided the original work is properly cited. 


\section{Excitation-energy sorting}

In fission, the energy difference between the ground-state masses of the initial fissioning system and the final fission fragments, given by the $\mathrm{Q}$ value, and the initial excitation energy of the fissioning nucleus $E_{C N}^{*}$, ends up either in the total excitation energy (TXE) or in the total kinetic energy (TKE) of the fragments. In the present work, we consider low-energy fission with initial excitation energies $E_{C N}^{*}$ up to a few $\mathrm{MeV}$. In this $E^{*}$ regime we can assume that there is no neutron emission before scission. Therefore, the TXE is released by neutron evaporation and gamma emission from the fission fragments. We may distinguish three classes of energy at scission, which add up to the final TXE of the fission fragments:

(i) Intrinsic excitations by single-particle or quasi-particle excitations.

(ii) Deformation energy.

(iii) Collective excitations stored in normal modes.

The intrinsic excitation energy is the sum of the $E^{*}$ above the barrier height and the fraction of the difference in potential energy between saddle and scission that is dissipated into intrinsic excitations. The deformation energy ends up as part of the $E^{*}$ available when the fission fragments recover their ground-state deformations. The damping of collective excitations as for example bending and wriggling modes leads to additional $E^{*}$ in the fragments.

We assume that already somewhat before the scission configuration the two nascent fragments have acquired their individual properties concerning shell effects $[3,4]$ and pairing correlations [5] and can be treated as two well defined nuclei set in thermal contact through the neck. The division of intrinsic excitations can be derived when thermal equilibrium is assumed among the intrinsic degrees of freedom in each fragment. As said above, the nuclear level density at low $E^{*}$ is very well described by the constant-temperature formula (1) with a specific value of $T$ for each fragment. This leads to a very interesting situation for the two nascent fragments near the scission-point configuration: There is no solution for the division of intrinsic $E^{*}$ with $T 1=T 2$. As long as some excitation energy remains in the fragment with the higher temperature, its $E^{*}$ is transferred to the fragment with the lower temperature. That means, a process of $E^{*}$ sorting takes place where all $E^{*}$ accumulates in the fragment with the lower value of the $T$ parameter, while the other fragment looses its entire $E^{*}$. According to eq. (2) the heavy fragment generally has the lower $T$ and thus attracts all the $E^{*}$. This behaviour is unique. To our knowledge all other systems in nature reach thermal equilibrium with $T 1=T 2$ before the thermal energy of the hotter object is completely exhausted. The flow of $E^{*}$ from the hot fragment to the cold fragment can be seen as a way to maximize the number of occupied states or its entropy. The number of available states of the light nucleus is small compared to that of the complementary fragment. Therefore, the situation in which the light nucleus has part of the $E^{*}$ leads to a smaller entropy than the situation in which the entire $E^{*}$ is transferred to the heavy nucleus which has considerably more available states. Finally, we would like to stress that the energy-sorting mechanism concerns only the sharing of intrinsic $E^{*}$ at scission. After scission (when the two fragments are not in contact anymore) the completely cold (generally light) fragment gains $E^{*}$ as it snaps back to its ground state deformation and through the damping of collective modes. We have thoroughly described the energy sorting process in Ref. [6]. It is also one of the ingredients of the GEF code [7,8].

\section{Neutron yields as a function of the fragment mass}

It is of great interest to find experimental signatures that reflect the excitation-energy sorting. These signatures must be able to distinguish between the intrinsic $E^{*}$ accumulated in each fission fragment. The number of evaporated neutrons as a function of the fragment mass is directly proportional to the $E^{*}$ of the fragment - except an offset of a few $\mathrm{MeV}$ that is taken away by gamma emission - and therefore should clearly reflect the peculiar situation of the full transfer of the intrinsic $E^{*}$ to the cold fragment. The neutron-induced fission of ${ }^{237} \mathrm{~Np}$ has been studied very carefully at two different neutron energies [9]. Fig. 1 shows the average number of evaporated neutrons as a function of the fragment mass $v(A)$. The well known saw-tooth-like behavior of this curve is attributed to the deformation 


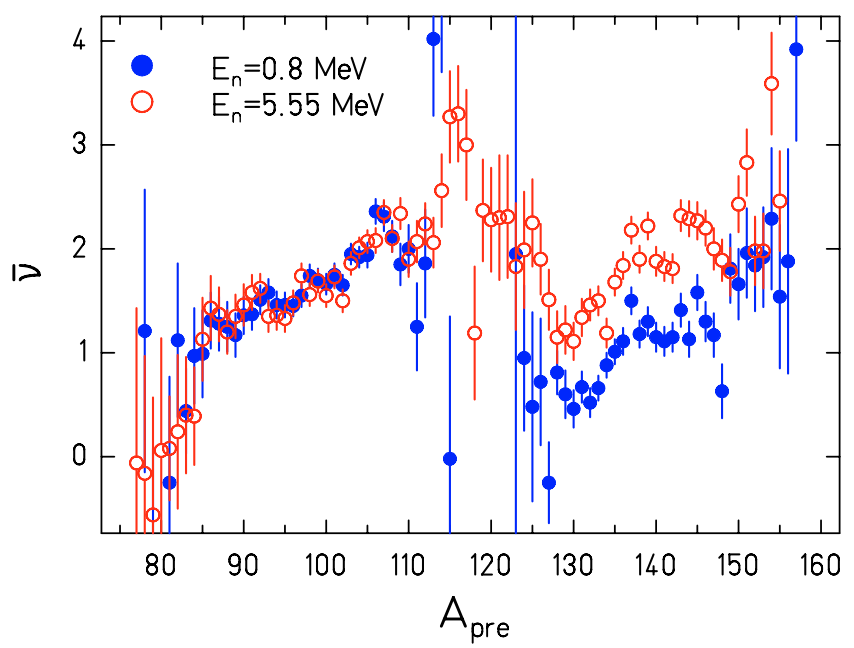

Fig. 1. Average number of prompt neutrons as a function of the primary fragment mass for the neutron-induced fission of ${ }^{237} \mathrm{~Np}$, data taken from Ref. [9].

energy. For asymmetric mass splits we observe a very peculiar feature: Fig. 1 shows that the increase of $E^{*}$ leads to an increase of the number of evaporated neutrons for the heavy fragment, only. Actually, a quantitative analysis of the data reveals that all of the increased $E^{*}$ appears in the heavy fragment. This observation is rather general as it was also found for other fissioning systems such as ${ }^{233} \mathrm{U}$ and ${ }^{238} \mathrm{U}$ and other incident particles like protons [10-13]. However, no clear explanation has yet been found for this effect. Actually, this is a direct consequence of the different constant temperatures of the two fragments at scission. According to eq. (2), the temperature of the heavy fragment, in the absence of strong shell effects, is always lower than the temperature of the light fragment. Therefore, the heavy fragment will absorb the entire available intrinsic $E^{*}$ and evaporate more neutrons. We would like to stress that our argumentation is based on the same assumptions as other work that investigates the sharing of intrinsic $E^{*}$ at scission [14-16]. That is, we have assumed independent fission fragments and thermal equilibration between the fragments at scission. What is substantially different is that we use the constant-temperature level density which correctly describes the behaviour of nuclei at moderate $E^{*}$ and not the commonly used Fermi-gas level density [17] which is only valid at high $E^{*}$.

\section{Even-odd effect in fission-fragment element yields}

At the end of the energy-sorting process the heavy fragment can gain additional $E^{*}$ if few nucleons are transferred through the neck so that the light fragment becomes even-even. The gain in $E^{*}$ can be up to four times the pairing gap. Therefore, according to the energy-sorting mechanism, there will be a tendency for the hot (normally the light) fragment to be fully paired. Let us now consider the dynamics of the energy-sorting process. The time $t$ to form a fully paired light fragment is the sum of the time needed for the light fragment to transfer all its $E^{*}$ to the heavier one, which we will call $t_{E^{*}}$, and the time to exchange few nucleons through the neck $t_{\text {exch }}$. Time $t_{\text {exch }}$ is rather short so that the time $t$ is dominated by $t_{E^{*}}$. The latter time will increase with the initial excitation energy in the light fragment $E_{0, \text { light }}^{*}$ since it will take a longer time to transfer all the energy from the light to the heavy nucleus. We consider that the initial excitation energy $E_{0, \text { light }}^{*}$ is proportional to the available excitation energy at scission $E_{s c i}^{*}$ which, as was said above, is the sum of the excitation energy at saddle $E_{C N}^{*}$ and the dissipated energy between saddle and scission $E_{s a d-s c i}^{*} . E_{C N}^{*}$ increases with beam energy and $E_{s a d-s c i}^{*}$ with the Coulomb parameter $Z^{2} / A^{1 / 3}$ since the saddle to scission path becomes longer [18]. For the systems investigated in Fig. 2 the Coulomb parameter is strongly correlated with the mass of the nucleus, thus it follows that $E_{s a d-s c i}^{*}$ increases with the nucleus mass. On the other hand, the time $t$ will decrease when the temperature difference $T 1-T 2$ between the two fragments increases. A higher 

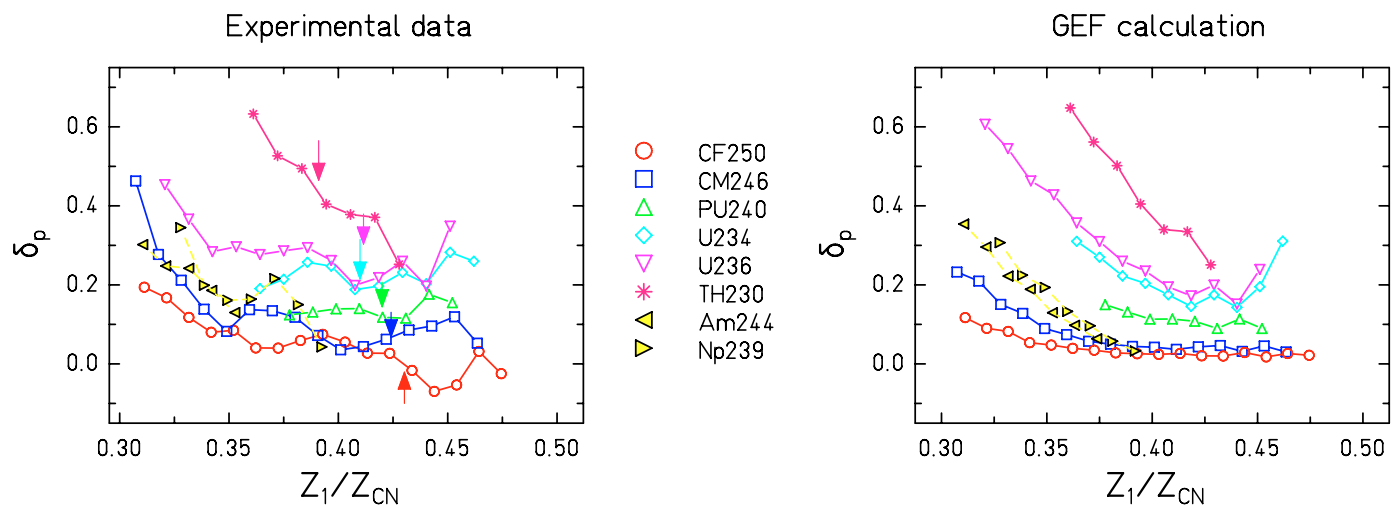

Fig. 2. Local even-odd effect as a function of asymmetry for various fissioning nuclei. The experimental data are shown on the left and the GEF calculation $[7,8]$ on the right.

temperature gradient leads to a faster flow of $E^{*}$ between the two fragments. According to eq. (2) an increase in temperature difference corresponds to an increase in the asymmetry of the mass split. To resume, the time $t$ follows the expression:

$$
t \propto \frac{E_{s c i}^{*}}{T 1-T 2} .
$$

As a consequence, $t$ will increase with the beam energy and the mass of the fissioning nucleus and will decrease with increasing asymmetry of the mass split. Let us now assume that there exists a time $t_{p}$ above which the exchange of protons through the neck is very much hindered ${ }^{1}$. If $t>t_{p}$, no net even-odd effect is induced because protons cannot be transferred to the heavy nucleus. Thus, according to the energy-sorting process the even-odd effect as a function of asymmetry should have a threshold character. The threshold asymmetry where the even-odd effect created by the energy sorting sets in (corresponding to the asymmetry for which $t=t_{p}$ ) will increase with the mass of the fissioning nucleus. This is in good agreement with the experimental systematics on the even-odd effect presented in Refs. [20,21]. The left part of Fig. 2 shows experimental data on the local even-odd effect as a function of charge asymmetry for different fissioning nuclei. The data have been taken from Ref. [22]. It presents data measured at ILL where fission was induced with thermal neutrons. The experimental data illustrate clearly how the local even-odd effect decreases with increasing mass of the fissioning nucleus. For a fixed even-Z fissioning nucleus, the general trend presented by the data is a small and rather constant even-odd effect close to symmetry and a strong increase as we move to more asymmetric fission. The threshold asymmetry increases with the mass of the fissioning nucleus. For ${ }^{230}$ Th the threshold character is not shown by the data. However, we presume that this is because the threshold asymmetry for this nucleus is close to symmetry where no data have been measured. It is interesting to note that the even-odd effect of the odd-Z fissioning systems ${ }^{244} \mathrm{Am}$ and ${ }^{239} \mathrm{~Np}$ follows the general behavior of the even- $Z$ fissioning systems. Their values are very close to those of the neighboring even-Z systems. For several systems, the data point in Fig. 2 that is closest to symmetry is appreciably higher than expected from the global trend. This effect may be associated to the influence of the $\mathrm{Z}=50$ shell in the complementary fragment, which is known to enhance the yield of tin isotopes and, thus, leads to a local increase of the deduced even-odd effect. In the GEF code $[7,8]$ the dependence of the local even-odd effect with asymmetry is modeled with a Gauss-integral. The threshold asymmetry value (the value at which the Gauss-integral is $1 / 2$ ) is the one that full fills the condition $C *\left(E_{s c i}^{*} /|T 1-T 2|\right)=t_{p}$, where $C$ is a constant. $C / t_{p}$ is adjusted to the data and has a value of 0.035 for all nuclei. The width of the Gauss function is set proportional to $|T 1-T 2|$. The

\footnotetext{
${ }^{1}$ This hindrance is due to the Coulomb barrier in the neck region which increases with the distance between the two fragments. The difference between the inner barrier for neutrons and protons is shown in Fig. 16 of Ref. [19]. The latter calculations have been done for two nuclei in contact during transfer reactions. However, we expect similar results for fission in spite of the slightly different shape of the scission configuration.
} 
scaling factor for $|T 1-T 2|$ is fitted to the experimental data. A value of $0.07 / \mathrm{MeV}$ is obtained for all the nuclei considered. On the right part of Fig. 2 the results of our model GEF for the same fissioning systems are presented. All the features mentioned above are nicely reproduced by our calculation. The energy-sorting mechanism also predicts that, for a given fissioning nucleus, the threshold asymmetry should increase with increasing beam energy. Unfortunately there are no data to verify this statement.

\section{Conclusions}

Moderately excited nuclei are peculiar systems where the temperature remains constant with increasing $E^{*}$. The very special feature of this phenomenon in nuclei is that the constant-temperature regime reaches down to zero energy. The scission configuration of the fission process offers the unique possibility to investigate how two different nuclei in this special regime of constant temperature share the available intrinsic excitation when they are in thermal contact. We have shown for the first time that the excitation energy keeps flowing from the hot to the cold fragment until the excitation energy of the hot fragment is completely exhausted. The $E^{*}$-sorting effect explains very easily why an increase in $E^{*}$ leads to an increase of the number of prompt neutrons of the heavy fragment, only. Indeed, the temperature of the heavy fission fragments is generally lower than that of the light ones. Therefore, all the intrinsic $E^{*}$ is cumulated in the heavy fragment. The energy-sorting process is also reflected by the dependence of the even-odd effect in fission-fragment element yields with mass asymmetry. This dependence has a threshold character with a threshold asymmetry that increases with beam energy and the mass of the fissioning nucleus. Unfortunately, there is still an important lack of data concerning $v(A)$ and the local even-odd effect as a function of asymmetry. It would be extremely interesting to measure precisely the evolution of these two quantities with $E^{*}$. In that way we could investigate for example up to which $E^{*}$ the energy-sorting mechanism survives.

This work was supported by the EURATOM 6. Framework Programme "European Facilities for Nuclear Data Measurements" (EFNUDAT), contract number FP6-036434. We thank F. Kaeppeler for providing us with the numerical values of the neutron-yield data.

\section{References}

1. T. von Egidy and D. Bucurescu, Phys. Rev. C 72, (2005) 044311.

2. A. V. Voinov et al., Phys. Rev. C 79, (2009) 031301(R).

3. U. Mosel and H. Schmitt, Phys. Rev. C 4, 2185 (1971).

4. J. Maruhn and W. Greiner, Z. Phys. 251, 431 (1972).

5. H. J. Krappe and S. Fadeev, Nucl. Phys. A 690, 431 (2001).

6. K.-H. Schmidt and B. Jurado, Phys. Rev. Lett. 104, (2010) 212501.

7. K.-H. Schmidt, B. Jurado, these proceedings.

8. www.cenbg.in2p3.fr/GEF

9. A. A. Naqvi et al., Phys. Rev. C 34, (2010) 212501.

10. S. C. Burnett et al., Phys. Rev. C 3, (1971) 2034.

11. C. J. Bishop et al., Nucl. Phys. A 150, (1970) 129.

12. R. Mueller et al., Phys. Rev. C 29, (1984) 885.

13. M. Strecker et al., Phys. Rev. C 41, (1990) 2172.

14. D. G. Madland and J. R. Nix, Nucl. Sci. Eng. 81, 213 (1982).

15. S. Lemaire et al., Phys. Rev. C 72, (2005) 024601.

16. N. V. Kornilov, et al., Nucl. Phys. A 789, 55 (2007).

17. H. A. Bethe, Phys. Rev. 50, (1936) 332.

18. M. Asghar, R. W. Hasse, J. Phys. C 6, (1984) 455.

19. W. von Oertzen and A. Vitturi, Rep. Prog. Phys. 64, 1247 (2001).

20. M. Caamaño et al., to be published, arXiv:0909.1059v1 [nucl-ex].

21. F. Rejmund et al., these proceedings.

22. F. Rejmund et al., GANIL P 2010-01, http://hal.in2p3.fr/in2p3-00487186/fr/. 